\title{
Multicell Downlink OFDM Subchannel Allocations Using Dynamic Intercell Coordination ${ }^{1}$
}

\author{
Mahmudur Rahman and Halim Yanikomeroglu \\ Broadband Communications and Wireless Systems (BCWS) Center \\ Department of Systems and Computer Engineering \\ Carleton University, Ottawa, Ontario K1S 5B6, Canada \\ \{mmrahman,halim\}@sce.carleton.ca
}

\begin{abstract}
In this paper, we present a novel integer programming (IP) optimization formulation for downlink OFDM subchannel allocations in a multicell system and propose viable sub-optimal solutions. The schemes use dynamic intercell coordination in order to avoid or minimize dominant co-channel interference and achieve maximum network throughput by exploiting multicell multiuser diversity and interference avoidance gains. The performance of the proposed schemes is compared with that of reference round-robin schedulers without intercell coordination. It is observed from snapshot simulation results that the proposed schemes outperform the reference schemes in terms of throughput and quality of service (QoS) considerations.
\end{abstract}

Index Terms- OFDM resource allocation, multicell diversity, interference avoidance, radio resource optimization.

\section{INTRODUCTION}

Orthogonal frequency division multiplexing (OFDM) has been considered as a promising modulation technique for the future generation wireless systems, such as IEEE 802.16a/e and WINNER [1], not only for its inherent ability to combat inter-symbol interference (ISI) resulting from frequency selective fading, but also for the flexibility it offers in radio resource allocations as each subcarrier can be modulated adaptively to exploit frequency-domain diversity and improve achievable data rates on available frequency spectrum.

In a single cell environment, downlink multiuser diversity is achieved by allocating subcarriers for transmissions to user terminals (UTs) where it can be utilized best depending on the channel conditions. Numerous studies are available in the literature that investigate this multiuser diversity gain using various optimization techniques [2]-[4]. Computational complexity is an inherent concern in any optimization problem, and in order to alleviate such burden, different sub-optimal approaches are proposed [5],[6].

Most of the available studies consider single cell networks; however in designing practical networks, optimization should be performed in a multicell environment considering one of the most performance limiting factors, namely intercell interference. Only a few research works on multicell allocation can be found in the literature [7],[8]. A lin- -ear programming (LP) formulation is proposed in [7] to obtain maximized network throughput, where subchannels are partitioned and assigned fixed reuse factors such that user terminals at the cell edge can use subchannels with higher reuse factors. In [8], separate algorithms are used at the radio network controller (RNC) and base stations (BSs) in order to optimize resources in longer and shorter time-scales in a semidistributed fashion. Subchannels are allocated to each BS by the RNC based on mutual interference situations and traffic demands, and fading diversity is achieved through local allocations by the BS.

A chunk is defined as a collection of consecutive subcarriers over a defined time period, which is regarded in this paper as the minimum granularity of radio resource unit. In our research, we formulate the multicell subchannel (or chunk) allocation problem by using integer programming method to investigate feasible sub-optimal solutions. The proposed schemes differ from [7] in that resulting reuse of resources in our schemes is dynamic and hence no cell planning or reuse partitioning is required. Also, as opposed to [8], the proposed schemes in this paper address the so called chain effect of interferers that arise as a consequence of clustering BSs by including factors in optimization formulation.

The objective of the proposed schemes is to achieve multicell multiuser diversity as well as intercell interference avoidance gains. In particular, the proposed schemes result in dynamic reuse factor for each chunk in a group of neighboring cells to provide maximum possible spectral efficiency given instantaneous mutual interference among neighboring cells. Intercell coordination is an integral part of the proposed schemes to ensure appropriate treatment of potential dominant intercell interference.

The proposed schemes work in a semi-distributed architecture as follows. A number of mutually interfering neighboring cells (due to relative locations, antenna directivities, and gains) are assumed to form a dominant interferer group. The in-group cells dynamically coordinate with each other to yield optimum reuse of resources given mutual interference situation. A logical node (i.e., RNC) executes computational tasks of the allocations and informs the BSs about resource allocation decisions.

The performance of the proposed schemes is compared

\footnotetext{
${ }^{1}$ This work was supported by the Natural Sciences and Engineering Research Council (NSERC) of Canada under participation in the Wireless World Initiative New Radio (WINNER) project - www.ist-winner.org.
} 
with that of the reference schemes in which intercell coordination is not used and resource allocations are performed in a round-robin fashion. The rest of this paper is organized as follows. The optimization formulation along with brief descriptions of the system under study is presented in Section II. Section III describes simulation models and parameters. Results from snapshot simulations are discussed in Section IV. Section V addresses the implementation complexity issues of the proposed schemes followed by the conclusions in Section VI.

\section{Formulation of the Optimization Problem}

Let us consider a downlink frequency division duplex (FDD) OFDM system where $i, j$, and $k$ are the indices for chunks, UTs, and BSs, respectively. A UT can be assigned multiple chunks in order for it to satisfy the required data rate.

Fig. 1 shows the layout of an example network where each cell site is composed of three hexagonal sectors. Each sector is equipped with $120^{\circ}$ directional transmit antenna. The available spectrum is equally divided among three sectors and is reused in every cell site. The intensity of the shadings in the figure represents overlapping sub-bands of spectrum.

For the darkest shaded sectors in Fig. 1, it is seen that downlink transmissions from BS 1 would be potential dominant interference to the UTs in BS 2, due to relative locations and antenna directivities. Therefore, for a given chunk $i$, the questions are (1) should chunk $i$ be masked in $B S$ 2 when it is used at BS 1? (2) should BS 2 use it instead and $B S 1$ masks as BS 2 utilizes the chunk more efficiently? or, (3) should both $B S 1$ and 2 use chunk $i$ concurrently, as the mutual interference permits to achieve better aggregate spectral efficiency than (1) or (2)? This is a combinatorial optimization problem which can be solved using binary integer programming.

The proposed optimization schemes answer the above questions by choosing the best possible option from those mentioned above. For the given network, BSs 1 and 2 form an interferer group, and they observe and forward mutual interference information to the RNC from time to time. Frequency of this information reporting depends on the channel coherence time. With similar arguments, BSs 2, 3, and 4 should belong to another interferer group, as UTs in BS 3 receives potential dominant interference from BSs 2 and 4. In a straightforward manner, it can be seen that BSs 3,4 , and 5 belong to a third interferer group, and BSs 5 and 6 to a fourth. A careful observation into the layout reveals that this pattern of interferer groups repeats in the layout for the given assumptions of system parameters and resource partitioning. Chunk allocations to these four groups can be optimized at the $\mathrm{RNC}$ as shown in Fig. 2. We describe the optimization formulations of the proposed schemes as follows.

Let us denote $\rho_{i, j, k \mid l}^{g}$, binary integer variable, to be the assignment indicator which takes a value of 1 if chunk $i$ is assigned to UT $j$ in BS $k$ of group $g$, given that a combination of other in-group BSs $l$ has been using chunk $i$ concurrently,

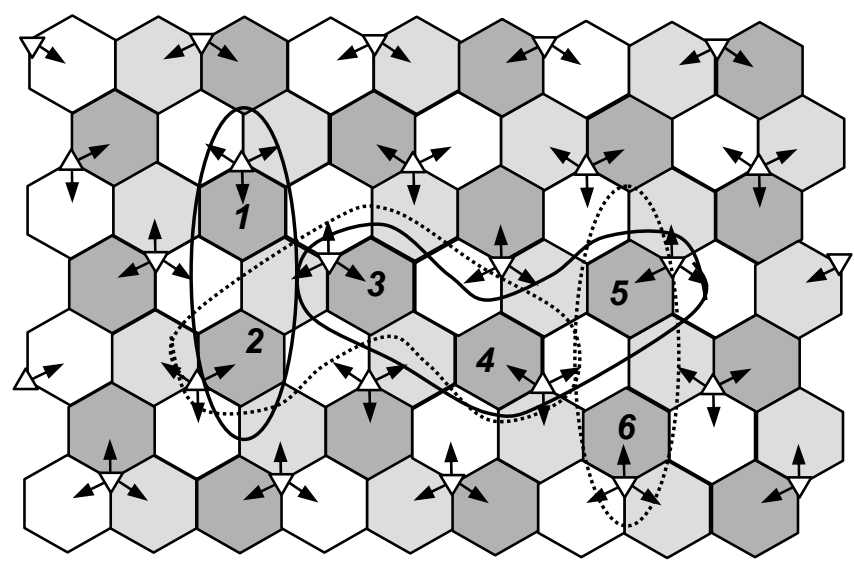

Fig. 1: Example network consisting of 3-sector cell sites

otherwise it is equal to zero. For instance, $\rho_{i, j, 3 \mid 2}^{2}=1$ indicates that chunk $i$ is assigned to UT $j$ in BS 3 of group 2, while the same chunk is also being used by other 2 in-group BSs (BSs 2 and 4 in this case). Note that $l=0$ means BS $k$ is using the chunk alone in the group. The superscript $g$ is used for convenience to account for the fact that a particular BS can be a member of different interferer groups.

As power control does not yield significant performance gain in OFDM system [3] compared to the complexity it adds to system's operations, we consider equal power allocation to chunks. The objective of the proposed scheme is to maximize total number of loaded bits in all chunks and over all groups satisfying user data rate requirements at any resource allocation instant, which can be formally stated as below.

\section{Maximize}

$$
\sum_{i} \sum_{j} \sum_{k} \sum_{l} \sum_{g} \rho_{i, j, k \mid l}^{g} C_{i, j, k \mid l}^{g}
$$

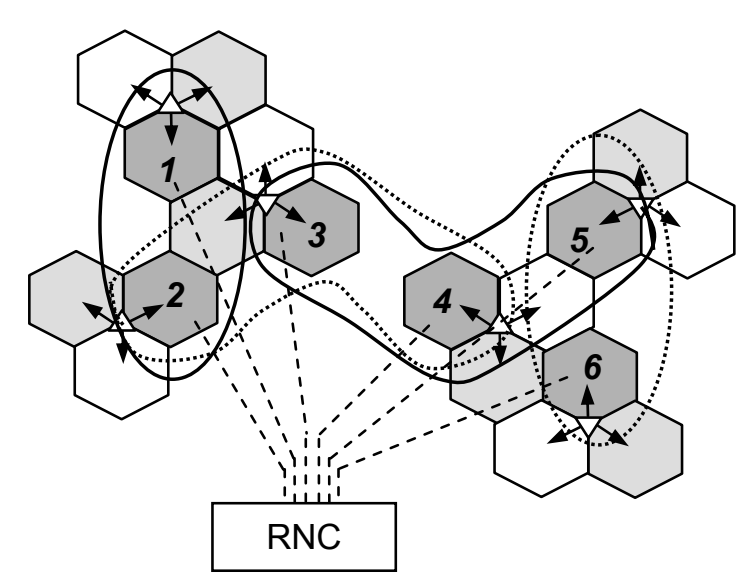

Fig. 2: Interferer groups $\{1,2\},\{2,3,4\},\{3,4,5\}$, and $\{5,6\}$ considering the darkest shaded ${ }^{2}$ co-channel sectors

\footnotetext{
2 Only the darkest shaded sectors are considered in simulations.
} 
subject to

$$
\begin{aligned}
& \sum_{i} \sum_{l} \sum_{g} \rho_{i, j, k \mid l}^{g} C_{i, j, k \mid l}^{g} \geq R_{j, k} ; \quad \forall \\
& \sum_{j} \sum_{k \mid l=0} \rho_{i, j, k \mid l=0}^{g}=L_{0}^{g}, \\
& \sum_{j} \sum_{k \mid l=1} \rho_{i, j, k \mid l=1}^{g}=N_{1}^{g} L_{1}^{g}, \\
& \left.\begin{array}{l}
\sum_{j} \sum_{k \mid l=2} \rho_{i, j, k \mid l=2}^{g}=N_{2}^{g} L_{2}^{g}, \\
\bullet \sum_{j} \sum_{k \mid l=M} \rho_{i, j, k \mid l=M}^{g}=N_{M}^{g} L_{M}^{g}, \\
\begin{array}{l}
L_{0}^{g}+L_{1}^{g}+\cdots+L_{M}^{g} \leq 1 ; \\
\sum_{j} \sum_{l} \sum_{g} \rho_{i, j, k \mid l}^{g} \leq 1
\end{array} \quad ;(i, g)
\end{array}\right\}
\end{aligned}
$$

$$
\left.\begin{array}{ccc}
\sum_{l} \rho_{i, j, k \mid l}^{g_{1}} C_{i, j, k \mid l}^{g_{1}} \sum_{l}^{\sum_{l} \rho_{i, j, k \mid l}^{g_{1}}=0, \sum_{l} \rho_{i, j, k \mid l}^{g_{2}}=1} & g_{1} \neq g_{2} \\
\sum_{i, j, k \mid l}^{g_{2}} C_{i, j, k \mid l}^{g_{2}} & \underbrace{<1}_{\sum_{l} \rho_{i, j, k \mid l}^{g_{2}}=0, \sum_{l} \rho_{i, j, k \mid l}^{g_{1}}=1} & k=g_{1} \cap g_{2} \\
\forall(i, j, k)
\end{array}\right\}
$$

In the above formulation, $C_{i, j, k \mid l}^{g}$ denotes the number of bits loaded on chunk $i$ if it is allocated to UT $j$ in BS $k$ of group $g$, given that the same chunk is also being used by a combination of in-group BSs $l$. For different in-group interferer combinations, signal to interference plus noise ratio (SINR) can be predicted and $C_{i, j, k \mid l}^{g}$ can be calculated based on feasible adaptive modulation and coding (AMC) modes.

The constraints in (2) ensure that different data rate requirements for individual $\mathrm{UT}$ in all cells are satisfied. $R_{j, k}$ denotes the number of bits per chunk time duration required by $\mathrm{UT} j$ in BS $k$. A chunk could be used by all in-group cells or by a subset of in-group cells depending on the mutual interference situation, which is modeled by constraints in (3). Here, $L_{\mathrm{x}}$ is a binary integer, and $N_{\mathrm{x}}$ is an integer which denotes the number of in-group cells using a particular chunk concurrently. A cell can be a member of different interferer groups as shown in Fig. 2. Constraints in (4) enforce that a chunk can only be used once in a cell in order to avoid intracell interference irrespective of cell's association with different groups. A BS which is common in two different groups has to be vigilant about interference from both groups. Therefore, for a particular UT, the BS should consider the worse interferers from these two groups in selecting AMC modes; these constraints are expressed in (5).
Complexity of the integer programming increases exponentially as the number of variables and constraints increases. Therefore, sub-optimal solution to the above problem is required. In our approach, we partition the problem into a number of smaller problems and solve iteratively with reduced solver complexity. For example, a subset of unassigned chunks can be taken at a time to allocate optimally to a subset of rate unsatisfied UTs. The iteration terminates when the data rates of all UTs are satisfied or the resources are exhausted. This iterative approach is a tradeoff between the complexity and optimality of the solution; i.e. higher the number of chunks to be allocated at a time, better the achieved optimality. The detail of the sub-optimal solution is described in the pseudo codes in Fig. 3.

\section{Simulation Models and Parameters}

A cluster of six cells with four interferer groups shown in Fig. 2 is simulated to evaluate the proposed allocation schemes. The available spectrum of $45 \mathrm{MHz}$ in the $3.95 \mathrm{GHz}$ frequency band is equally divided among three sectors giving each sector a total of 384 subcarriers each $39.0625 \mathrm{KHz}$. The whole spectrum is available in each cell. A chunk comprising 8 consecutive subcarriers is considered as the smallest allocation unit. It is a time-frequency resource unit occupying $0.3456 \mathrm{~ms}$ and $312.5 \mathrm{KHz}$, which translates into $8 \times 12$ OFDM symbols [9]. We assume that minimum downlink data rate requirement for each UT is 2 Mbps (i.e., 691.2 bits per chunk time duration).

Time and frequency correlated Rayleigh channel samples are generated from power delay profile for WINNER wide area scenario [10]. The user mobility is assumed to be 70 $\mathrm{km} / \mathrm{hr}$. The sector radius is considered to be 300 meters. The following exponential path-loss $(L)$ model has been used [10],

$$
L=38.4+35.0 \log _{10}(d)[\mathrm{dB}]
$$

where $d$ is transmitter-receiver separation in meters.

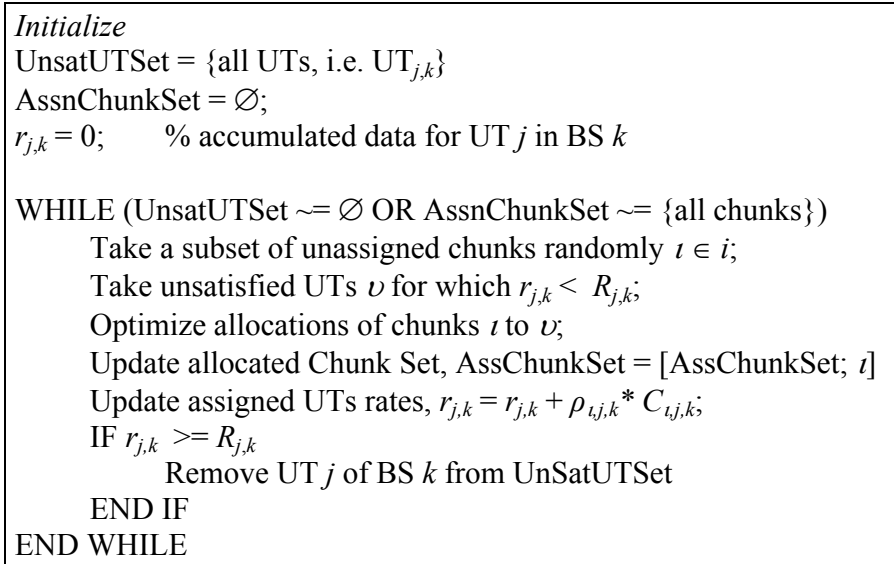

Fig. 3: Pseudo codes for sub-optimal solution 
The UT receive antennas are assumed to be omnidirectional, while the gain pattern for $120^{\circ}$ directional sector transmit antennas is considered as follows [10]:

$$
A(\theta)=-\min \left[12\left(\frac{\theta}{\theta_{3 d B}}\right)^{2}, 20\right][\mathrm{dB}],
$$

where the value of $\theta_{3 d B}$ is $70^{\circ}$ and $\theta$ varies from $-180^{\circ}$ to $180^{\circ}$.

The average thermal noise power is calculated with a noise figure of $5 \mathrm{~dB}$. We have considered independent lognormal random variables with a standard deviation of $8 \mathrm{~dB}$ for shadowing. Sector transmit power is assumed to be 39.81 Watts and chunks are assigned fixed equal powers.

AMC is used with bit interleaved coded modulation (BICM) as shown in Table I. The modulation options listed in the table are the mandatory AMC schemes available in the IEEE 802.16a standard [11]. A chunk using QPSK rate 1/2 can carry 96 information bits. Full queue model has been considered for downlink traffic.

Performance results have been observed for different network loadings ranging from 6 to 16 UTs per sector. For each loading scenario, a total of 100 allocation instants (in every $0.3456 \mathrm{~ms}$ ) are averaged to collect statistics.

Two different variations of round-robin schedulers have been considered as reference schemes. In the first, each UT is taken in a round robin manner, and assigned a set of downlink chunks starting from its best chunk until the data rate is satisfied. While in the second, UT is given one chunk at a time turn-by-turn until resources are exhausted.

As reference schemes do not employ intercell coordination, the predicted SINRs have to be determined conservatively based on the assumptions that all other cells are using the particular chunk concurrently.

In order to solve binary integer optimization problem, YALMIP [12] and LPSOLVE [13] have been used along with MATLAB. LPSOLVE is an integer linear programming (ILP) solver.

\section{Simulation Results}

The performance of the proposed scheme is evaluated based on the following observed parameters: average chunk reuse, average loaded capacity per chunk considering reuse, scheduled network capacity across all chunks, $50^{\text {th }}$ percentile received SINR on scheduled chunks, and percentage of users for which instantaneous data rate can not be satisfied on the allocation instant. We have simulated two different cases of the proposed scheme. One unassigned chunk is taken at a time in each iteration to optimally assign it to rate a unsatisfied UT in different cells in the first case (i.e. CASE 1), while a subset of 3 random unassigned chunks are taken at a time in the second case (i.e. CASE 2).

Fig. 4 compares the average number of concurrent use of chunks (i.e. mean reuse) for different schemes. Note that the averaging is performed on the used chunks only. The proposed schemes (CASE 1 \& CASE 2) show higher reuse of chunks
TABLE I

LOOKUP TABLE FOR AMC MODES ${ }^{3}$ BICM WITH BIT ERROR RATE OF $1.0 \times 10^{-4}$

\begin{tabular}{c|c|c}
\hline SINR Range $(\mathrm{dB})$ & AMC Mode & $\begin{array}{c}\text { Efficiency, } \eta \\
(\mathrm{Bits} / \mathrm{Sec} / \mathrm{Hz})\end{array}$ \\
\hline \hline $3.39 \leq \gamma<5.12$ & QPSK rate 1/2 & 1.0 \\
$5.12 \leq \gamma<6.02$ & QPSK rate 2/3 & 1.33 \\
$6.02 \leq \gamma<7.78$ & QPSK rate 3/4 & 1.5 \\
$7.78 \leq \gamma<9.23$ & QPSK rate 7/8 & 1.75 \\
$9.23 \leq \gamma<11.36$ & 16-QAM rate 1/2 & 2.0 \\
$11.36 \leq \gamma<12.50$ & 16-QAM rate 2/3 & 2.67 \\
$12.5 \leq \gamma<14.21$ & 16-QAM rate 3/4 & 3.0 \\
$14.21 \leq \gamma<16.78$ & 16-QAM rate 7/8 & 3.5 \\
$16.78 \leq \gamma<18.16$ & 64-QAM rate 2/3 & 4.0 \\
$18.16 \leq \gamma<20.13$ & 64-QAM rate 3/4 & 4.5 \\
$20.13 \leq \gamma<24.30$ & 64-QAM rate 7/8 & 5.25 \\
$\gamma \geq 24.30$ & 64-QAM rate 1 & 6.0 \\
\hline
\end{tabular}

even at lower loadings, as the objective function aims to maximize capacity return on chunk(s) taken in each iteration, given the mutual interference situations. On the other hand, the reference schemes are able to use only the good chunks at the lower loadings. It is observed from the figure that as the number of UTs increases, the reuse of chunks increases in the proposed and reference schemes. Optimization works better in a larger set of UTs in the proposed schemes; while the reference schemes need more and more chunks with less efficient AMC, as the number of UTs per sector increases. Consequently, reference schemes require higher chunk reuse compared to CASE 1 at 16 UTs per sector. Due to the snapshot nature of simulations performed in this study where shadowing effect is not averaged out well, the mean reuse results for the proposed schemes at 6 UTs per sector appear to be high. The reason is that the UTs are assigned good chunks with high reuse at this loading.

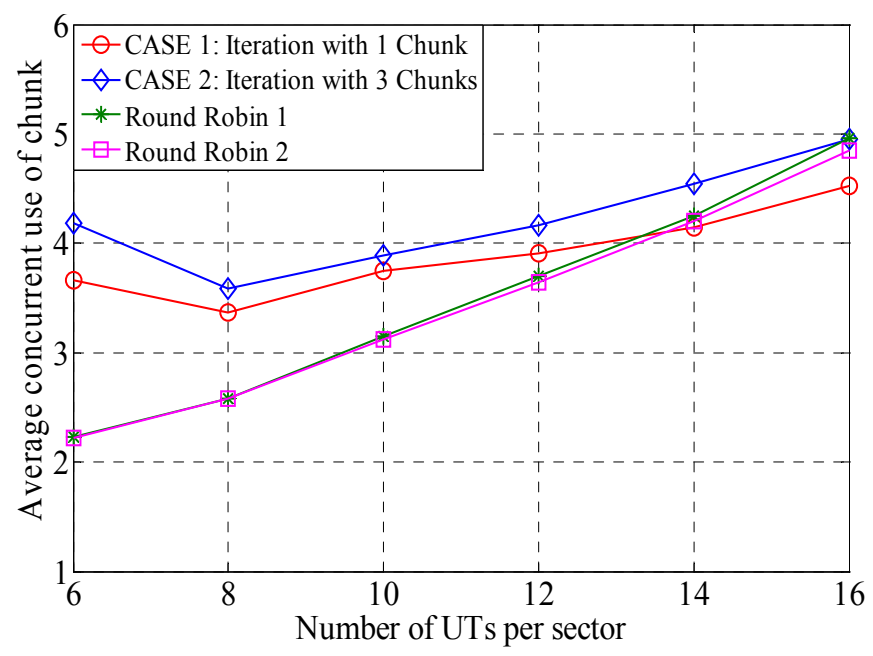

Fig. 4: Mean reuse of used chunks in the network

3 Data for the modulation curves are provided by Dr. Sirikiat Lek Ariyavisitakul. 
The mean reuse does not reflect the capacity gain completely as chunks use AMC; we refer to Fig. 5 where the average capacity achieved on each used chunk considering chunk reuse is shown. It is obvious that the capacity return on each used chunk in the proposed schemes would always be better than the reference schemes due to optimization. Also, as the number of UTs increases, optimized schemes yield improved returns which is again described by better multiuser diversity as for the mean reuse. Although the reference schemes show comparable mean reuse at 16 UTs per sector, they achieve $25 \%$ less average capacity per chunk compared to that in CASE 2. Because, AMC modes selected for chunks in the reference schemes are based on the conservative estimation of SINRs.

The average total capacity on all chunks (i.e. number of loaded bits per $0.3456 \mathrm{~ms}$ for all BSs) for different schemes is presented in Fig. 6. At 16 UTs per sector, the optimization scheme with 3 chunks (CASE 2) shows around 30\% and 16\% higher network capacity compared to those in the reference schemes and the scheme with 1 chunk (CASE 1), respectively. The gain in CASE 2 compared to CASE 1 is achieved from better optimality. It should be noted here that even better total capacity is expected if more chunks are taken to optimize at a time with the cost of higher solver complexity.

The instantaneous data rate of a UT can not be satisfied due to the following two reasons: 1) in this snapshot simulations, shadowing values are not changed; therefore if a UT is highly shadowed, it will see poor SINRs on most of its chunks 2) if there are not enough remaining good chunks for the UT due to prior allocations to other UTs. We are interested more on the second issue when BSs do not have resources and consequently UTs remain unsatisfied; this effect will be more visible at the higher network loadings. Percentage of UTs for which instantaneous data rate can not be satisfied is compared in Fig. 7. As a result of better capacity return on each chunk, the proposed schemes are able to support higher number of UTs per sector. It is noticed that the percentage of unsatisfied UTs in CASE 2 is a bit higher compared to that in CASE 1.

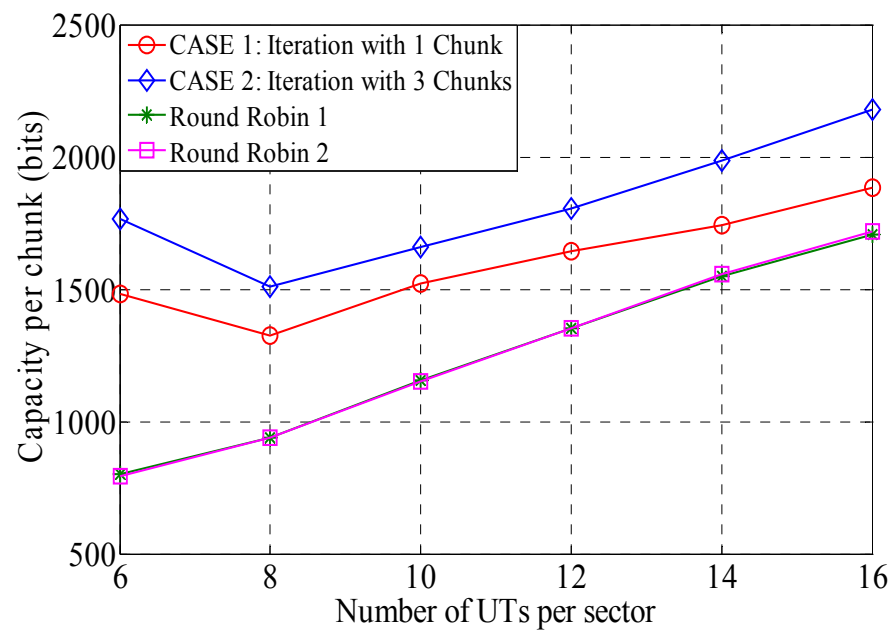

Fig. 5: Average capacity per chunk at the allocation instant considering reuse
This is due to the fact that some UTs in CASE 2 can be unfairly over-assigned resources as a result of optimization iteration with 3 chunks at a time. For instance, an unsatisfied UT that has already been assigned resources in earlier iterations but still needs little more to be satisfied may end up taking all three chunks in its final turn. As a result of this unfairness, other UTs may starve.

The $50^{\text {th }}$ percentile received SINRs on allocated chunks for different schemes are shown in Fig. 8. It is observed that the received SINRs in the reference schemes are better at the lower loading points. This is due to the fact that as reference schemes do not use coordination, estimated SINRs are determined conservatively assuming all BSs would use each chunk concurrently. Therefore at a low network loading, it is likely that the reference schemes would use only those chunks that experience minimum interference (and hence good SINR) resulting low chunk reuse. For instance in Fig. 4, reuse in the reference schemes is low at the lower loadings, therefore better received SINR is observed. However, as loading values increase more chunks are needed, and increased reuse in the reference schemes results in lower chunk SINRs. On the other hand, the proposed schemes show consistent SINRs as the reuse of chunks remains high at every loading point. Although the reference schemes experience similar SINR compared to that in CASE 2 at 16 UTs per sector, CASE 2 results much higher capacity due to intelligent allocations of chunks.

\section{IMPLEMENTATION COMPLEXITY ISSUES}

Two different kinds of complexities are involved with the proposed schemes; the solver complexity and the signaling complexity. The solver complexity of the integer programming method can be alleviated by partitioning the problem into smaller problems and solve iteratively for suboptimal solutions as proposed. The signaling overhead is related to the frequency of the channel reporting and resource allocation operations, which depends on the mobility of the UT and hence resulting channel coherence time. For example,

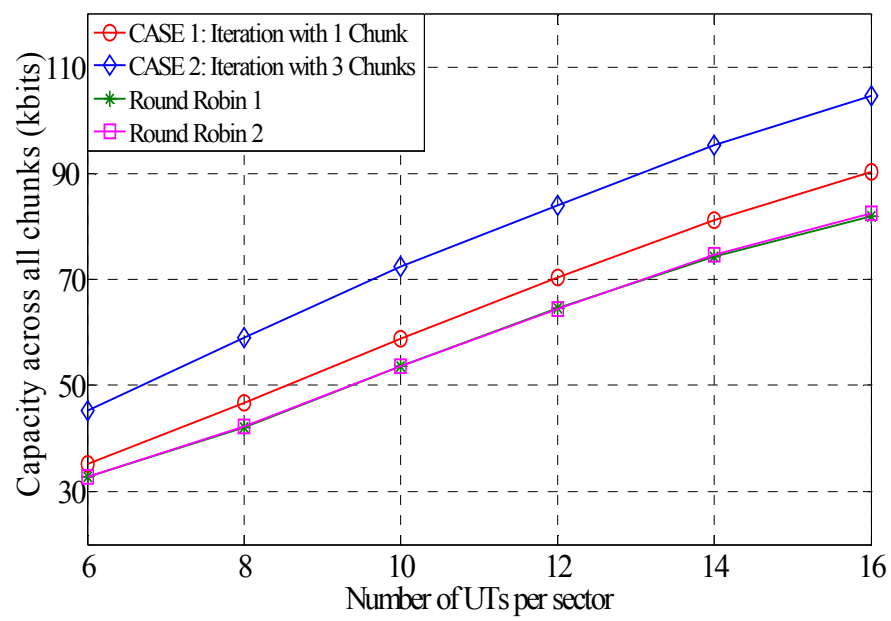

Fig. 6: Capacity on all chunks at the allocation instant 


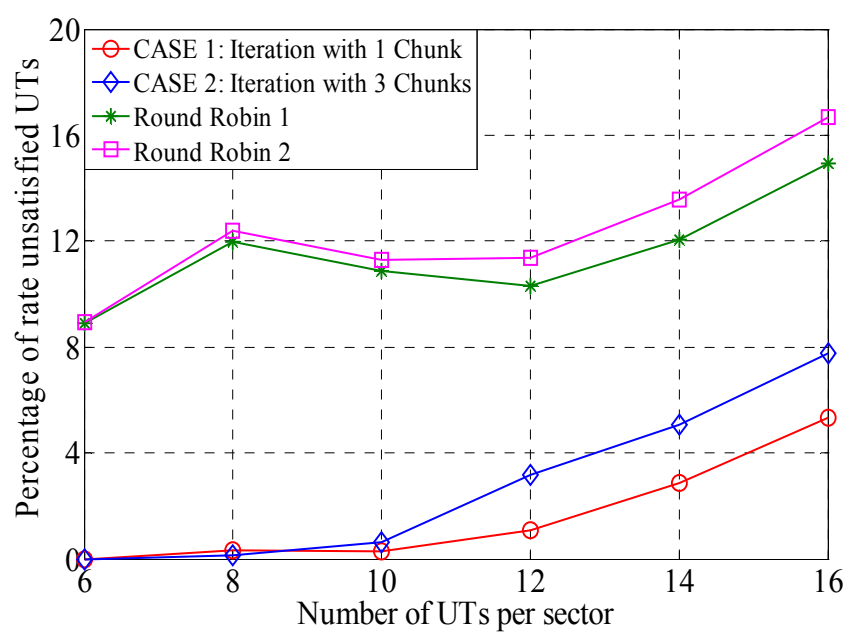

Fig. 7: Percentage of rate unsatisfied UTs

for a UT moving at $30 \mathrm{~km} / \mathrm{hr}$, channel coherence time would be around $4 \mathrm{~ms}$. Therefore, channel reporting and resource allocations have to be performed in every 12 chunks time duration. However for a speed of $70 \mathrm{~km} / \mathrm{hr}$, the frequency would be around 5 chunks time duration. Therefore, high signaling overhead would be required to support high mobility UTs. However, BS-RNC-BS signaling can be done using high data rate backbone connections such as fiber links.

\section{Conclusions}

A novel binary integer programming formulation for downlink multicell chunk allocation with dynamic intercell coordination is presented in this paper. The results of suboptimal solutions using iterative optimization for a subset of chunks at a time are compared with those of the reference round-robin schemes without intercell coordination. Our proposed schemes exploit the fact that a chunk can be severely interfered for one UT but can be quite good for another UT due to different shadowing, fading, and antenna directivities of the associated links. Therefore, there are potentials for high reuse of chunks with high returns if they are allocated intelligently. It can be concluded from observed simulation results that the proposed multicell allocation schemes outperform reference round robin schedulers in terms of scheduled throughput and number of supported UTs.

\section{ACKNOWLEDGEMENTS}

Authors are thankful to Mr. Feroz Bokhari for his review and useful comments on the manuscript.

\section{REFERENCES}

[1] Wireless World Initiative New Radio, www.ist-winner.org.

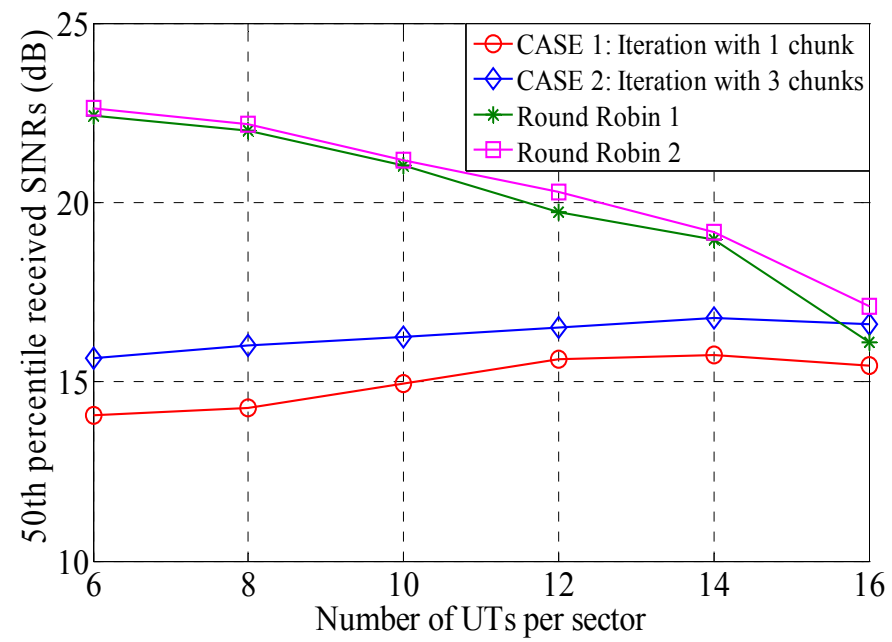

Fig. 8 : $50^{\text {th }}$ percentile received SINRs on scheduled chunks

[2] C.Y. Wong, R.S. Cheng, K.B. Letaief, and R.D. Murch, "Multiuser OFDM with adaptive subcarrier, bit, and power allocation," IEEE Journal on Selected Areas in Communications, vol. 17, no. 10, pp. 1747-1757, October 1999.

[3] W. Rhee and J.M. Cioffi, "Increase in capacity of multiuser OFDM system using dynamic subchannel allocation," in Proc. IEEE Vehicular Technology Conf., vol. 2, pp. 1085-1089, May 2000.

[4] H. Yin and H Liu, "An efficient multiuser loading algorithm for OFDM-based broadband wireless systems," in Proc. IEEE Global Telecommunications Conference (Globecom) 2000, San Francisco, USA, pp. 103-107, 2000.

[5] C.Y. Wong, C.Y. Tsui, R.S. Cheng, and K.B. Letaief, "A realtime sub-carrier allocation scheme for multiple access downlink OFDM transmissions," in Proc. IEEE Vehicular Technology Conference (VTC Fall) 1999, Amsterdam, Netherlands, pp. $1124-1128,1999$.

[6] I. Kim, H.L. Lee, B. Kim, and Y.H. Lee, "On the use of linear programming for dynamic subchannel and bit allocation in multiuser OFDM," in Proc. IEEE Global Telecommunications Conference (Globecom) 2001, San Antonio, USA, pp. 36483652, 2001.

[7] H. Kim, Y. Han, and J. Koo, "Optimal subchannel scheme in multicell OFDMA systems," in Proc. IEEE Vehicular Technology Conf., vol. 3, pp. 1821-1825, May 2004.

[8] G. Li and H. Liu, "Downlink dynamic resource allocation for multi-cell OFDMA systems," in Proc. IEEE Vehicular Technology Conf., vol. 3, pp. 1698-1702, October 2003.

[9] Test Scenarios and Calibration Cases Issue 2, WINNER Deliverable D6.13.7, December 2006, https://www.istwinner.org.

[10] Final report on link level and system level channel models, WINNER Deliverable D5.4, November 2005, https://www.istwinner.org.

[11] IEEE Std. 802.16a for Local and Metropolitan Area Networks, Air interface for fixed broadband wireless access systems, IEEE, April 2003.

[12] J. Löfberg, "YALMIP: a toolbox for modeling and optimization in MATLAB," In Proceedings of the CACSD Conference, Taipei, Taiwan, 2004.

[13] M. Berkelaar, K. Eikland, and P. Notebaert, lp_solve version 5.5, http://tech.groups.yahoo.com/group/lp_solve. 